Interfaces and Free Boundaries 12 (2010), 151-155

DOI $10.4171 / \mathrm{IFB} / 230$

\title{
Stable constant-mean-curvature hypersurfaces are area minimizing in small $L^{1}$ neighborhoods
}

\author{
FRANK MORGAN \\ Department of Mathematics and Statistics, Williams College, \\ Williamstown, MA 01267, USA \\ E-mail: Frank.Morgan@williams.edu \\ ANTONIO Ros \\ Departamento de Geometría y Topología, Facultad de Ciencias, Universidad de Granada, \\ 18071 Granada, Spain \\ E-mail:aros@ugr.es
}

[Received 3 December 2008 and in revised form 31 January 2010]

\begin{abstract}
We prove that a strictly stable oriented constant-mean-curvature hypersurface in a smooth closed manifold of dimension less than or equal to 7 is uniquely homologically area minimizing for fixed volume in a small $L^{1}$ neighborhood, proving a conjecture of Choksi and Sternberg.
\end{abstract}

2010 Mathematics Subject Classification: 49Q20, 53C42.

\section{Introduction}

By work of White $[\mathrm{W}]$ and Grosse-Brauckman $[\mathrm{Gr}]$, a strictly stable oriented constant-meancurvature hypersurface $S_{0}$ in a smooth ambient Riemannian manifold $M$ is minimizing in a small Riemannian distance neighborhood $U$ of $S_{0}$ among competitor hypersurfaces $S \subset U$ enclosing the same volume. Assuming $M$ compact, we extend their results to a small $L^{1}$ neighborhood of $S_{0}$, i.e., to hypersurfaces $S$ such that $S-S_{0}$ bounds a region with net volume 0 and small total volume.

Stable constant-mean-curvature hypersurfaces in $M$ appear in particular as solutions of the isoperimetric problem; see for instance [R1, R2].

If the ambient space is a flat 3-torus $T^{3}$ there is a connection between the isoperimetric problem and the study of mesoscale phase separation phenomena. For example, in diblock copolymers, different pieces of large molecules repel each other and, in an attempt to minimize the interfaces between such pieces, create a periodic structure. See Choksi and Sternberg [CS]. One simple model postulates periodic surfaces separating regions of fixed volume fraction and minimizing interface energy or area. A more sophisticated model with diffuse interfaces replaces a function which is 1 on one region and -1 on the other with a general $L^{1}$ function $u$ with fixed integral and minimizes the Cahn-Hilliard functional

$$
E_{\varepsilon}(u)=\int_{T^{3}}\left(\frac{\varepsilon^{2}}{2}|\nabla u|^{2}+W(u)\right) \mathrm{d} x,
$$

where $W$ is nonnegative with $W( \pm 1)=0$. The $W$ term encourages $u$ to focus on the values \pm 1 , while the $\nabla u$ term would minimize the transitions. So-called $\Gamma$-convergence theory shows that as $\varepsilon \rightarrow 0$, some subsequence of global minimizers of the Cahn-Hilliard energy converges to a sharp 
interface limit with $u= \pm 1$ of solutions to the isoperimetric problem (see [B, Example 0.1 and Comments p. 112], [CS, Prop. 3.1]). Conversely, Kohn and Sternberg [KS, Prop. 2.1 and §3.3] and Choksi and Sternberg [CS, Prop. 3.2] show by compactness that if a hypersurface $S_{0}$ minimizes area uniquely (up to translations) for given volume in an $L^{1}$-neighborhood, then there are nearby $L^{1}$-local minimizers of the Cahn-Hilliard energy $E_{\varepsilon}$ for small $\varepsilon$. Choksi and Sternberg [CS], p. 382 and Remark 7(ii)] conjecture that it suffices to assume $S_{0}$ has positive second variation. Our results prove this conjecture, providing local Cahn-Hilliard minimizers (Corollary 5). Pacard and Ritoré [PR, Thm. 4.2] use perturbation theory for PDEs to prove a similar result for nondegenerate critical points rather than local minima.

In flat 3-tori there are some beautiful minimal surfaces, the Schwarz $P$ and $D$ surfaces and the Gyroid $G$ of A. Schoen, which are closely related to complex phases appearing in periodic phase separation. Ross [Ro] has proved that these surfaces are stable for fixed volume and there is a particular interest in providing a mathematical treatment of these complex phases by minimizing locally the Cahn-Hilliard energy or other more sophisticated models. Corollary 5 proves nearby Cahn-Hilliard diffuse-interface versions of these surfaces.

For background in geometric measure theory see Giusti [G] and Morgan [M1].

\section{The proof}

Two homologous oriented hypersurfaces $S$ and $S^{\prime}$ in $M^{n}$ bound a region. Technically, this region is an $n$-dimensional integral current which is unique (up to multiples of $M$ in the case that $M$ is orientable). We define the $L^{1}$-distance $\left\|S-S^{\prime}\right\|_{L^{1}}$ between them as the minimum of the masses of the regions they bound. For example in $\mathbb{R}^{n}$ if $S$ and $S^{\prime}$ bound regions (of multiplicity +1$) \Omega$ and $\Omega^{\prime}$, respectively, then $\left\|S-S^{\prime}\right\|_{L}^{1}$ is just the volume of the symmetric difference between $\Omega$ and $\Omega^{\prime}$.
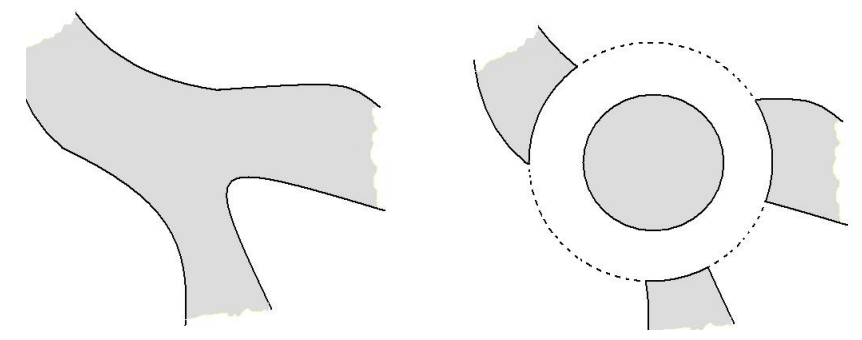

FIG. 1. Proof of the area growth estimate: $S$ at the left. At the right we have a competitor hypersurface which differs from $S$ inside a ball of radius $r$ and encloses the same volume as $S$ in this ball.

We will need the following isoperimetric version of the classical result after Fleming [F, Sect. 5] that for $n \leqslant 7$, area-minimizing hypersurfaces in the $\mathbb{R}^{n}$ are hyperplanes. For $n=3$ da Silveira ([dS], see also [LR] ) proved the result under the weaker hypothesis that $S$ be stable for fixed volume, i.e., in competition with surfaces which together with $S$ bound net oriented volume 0 .

PROPOSITION 1 Let $S$ be an oriented hypersurface (multiplicity one) without boundary in $\mathbb{R}^{n}$, $n \leqslant 7$, area-minimizing for fixed volume under changes of compact support. Then $S$ is either a hypersphere or a hyperplane.

Proof. If $S$ is compact, $S$ is a hypersphere by the standard isoperimetric inequality. Assuming $S$ is not compact, the hypothesis of the proposition implies that $S$ has constant mean curvature and is 
stable for fixed volume. Given $r>0$, inside the ball $B(r)$ around a fixed point of $S$, replace the region bounded by $S$ by a ball $B(\rho), 0 \leqslant \rho \leqslant r$, of the same volume as in Figure 1 . The resulting area inside $B(r)$ is at most twice the area of the hypersphere $\partial B(r)$. Hence the original area inside the ball of the minimizer $S$ is at most $C r^{n-1}$, for some $C$. Since this holds for all $r>0$, by Cheung $[\mathrm{C}]$ the hypersurface $S$ has mean curvature 0. By monotonicity of the mass ratio [A, Cor. 5.1(3) p. 446], the area divided by $\alpha_{n} r^{n-1}$, where $\alpha_{n}$ is the volume of the unit ball in $\mathbb{R}^{n-1}$, is nondecreasing in $r$, varying from 1 as $r$ approaches 0 to a limit $C_{0}$ as $r$ approaches infinity. Therefore homothetic contractions, restricted to balls about the origin, have area bounded below and above, so that by compactness [M1, 5.5 and remark p. 88], a subsequence converges to a nonzero limit, which has constant area ratio $C_{0}$ and is therefore a cone [A, Cor. 5.1(2)]. Since the cone minimizes area for given volume and $n \leqslant 7$, by regularity [M2] the cone must be a hyperplane (with multiplicity 1 because it is the boundary of a region) and $C_{0}=1$. Hence likewise $S$ has constant mass ratio 1 and must be a hyperplane.

Now we prove our main result.

THEOREM 2 In a smooth closed Riemannian manifold of dimension $n \leqslant 7$, let $S_{0}$ be a smooth oriented constant-mean-curvature hypersurface, possibly with boundary, with positive second variation for fixed volume and boundary. Then $S_{0}$ is uniquely homologically area minimizing for fixed volume among oriented hypersurfaces in a small $L^{1}$ neighborhood.

In particular, if $S_{0}$ bounds a region, then it minimizes area among hypersurfaces $S$ enclosing the same volume with $\left\|S-S_{0}\right\|_{L^{1}}$ small. It is not necessary to assume that $S_{0}$ is a boundary. Our proof gives that $S_{0}$ minimizes among competitors $S$ such that $\partial S=\partial S_{0}$ and $S-S_{0}$ bounds net oriented volume 0 .

Proof. Denote area, volume, and mean curvature by $A, V$, and $H$. The subscript 0 refers to $S_{0}$. Our hypersurface $S_{0}$ has positive second variation under smooth variations which fix volume (or equivalently under smooth variations which fix volume to first order). By Grosse-Brauckmann [Gr, Lemma 5], for some $C>0, S_{0}$ has positive smooth second variation for the energy

$$
F=A+H_{0} V+(C / 2)\left(V-V_{0}\right)^{2}
$$

under general smooth variations. As Grosse-Brauckman [Gr, last paragraph] points out, [W] Thm. 3] applies to show that $S_{0}$ uniquely minimizes $F$ in a neighborhood. To see this, let $\omega$ be a smooth differential form which over homologous surfaces gives the volume enclosed by $S_{0}$, such that $C \omega$ is small in a neighborhood of $S_{0}$ [W] end of Intro.]. To apply [W, Thm. 3], take $F$ to be the area integrand, $F_{1}=F+C \omega, F_{2}=F$, and $\phi(x, y)=(x-y)^{2} / 2 C$. By [W] Thm. 3], $S_{0}$ uniquely minimizes $F$ in a small neighborhood $U$ of its support. In particular, for fixed volume, $S_{0}$ uniquely minimizes $A$ in $U$.

To obtain a contradiction, suppose that there is a sequence of surfaces $S_{i}$ of no more area than $S_{0}$ converging in $L^{1}$ to $S_{0}$ and enclosing net signed volume 0 with $S_{0}$. Because the ambient manifold is compact, we may assume that $S_{i}$ minimizes area for fixed $\left\|S_{i}-S_{0}\right\|_{L^{1}}=\varepsilon_{i} \rightarrow 0$. On the complement of $S_{0}, S_{i}$ has two parts: where the region $\Omega_{i}$ bounded by $S_{0}-S_{i}$ has positive or negative orientation. (If $S_{i}$ and hence $\Omega_{i}$ has multiplicity, both decompose into pieces of multiplicity 1 [M1, Fig. 10.1.1].) Each part minimizes area for fixed volume; therefore $S_{i}$ is a smooth constant-meancurvature surface [M2, Cor. 3.7] (although the constants on the two parts need not be equal; we assert no regularity at points of $S_{0}$ ). By the first paragraph of this proof, each $S_{i}$ strays outside $U$. 
By replacing $S_{i}$ by a subsequence, we may assume that each $S_{i}$ strays outside of $U$ always with the same part or always with both parts. Hence by monotonicity, for a relevant part of $S_{i}$, the curvature of the sequence $S_{i}$ is not bounded in $M-U$. Indeed, if the mean curvature were bounded, then by monotonicity of the mass ratio [A Cor. 5.1(3) p. 446 and Remark 4.4], the area of $S_{i}$ outside a smaller neighborhood $U^{\prime}$ would be bounded below by some positive constant $\delta$, and then

$$
A\left(S_{0}\right) \leqslant \liminf A\left(S_{i}\right)-\delta \leqslant A\left(S_{0}\right)-\delta
$$

the desired contradiction.

Choose a point outside of $U$ on a relevant part of $S_{i}$ of maximum $|I I|^{2}$ (the sum of the squares of the principal curvatures) and scale the picture to make $|I I|^{2}=1$. A limit is minimizing for fixed volume in $\mathbb{R}^{n}$ and hence must be a round sphere by Proposition 1 . Hence for some large $i, S_{i}$ includes a small, nearly round sphere partly outside $U$. We may assume that there are no other points of that part of $S_{i}$ outside $U$, since otherwise we could repeat the argument on $S_{i}$ minus the first sphere and obtain a second such sphere, while replacing them with one sphere would do better. Hence in each part of $S_{i}$, there is at most one such sphere partly outside $U$. For a constant $c_{n}$ depending only on the dimension $n$, the total area and volume of such spheres satisfy $a>c_{n} v^{(n-1) / n}$.

Let $T_{i}$ be $S_{i}$ minus such spheres, so that $T_{i}$ lies in the neighborhood $U$ of $S_{0}$. Now

$$
F\left(T_{i}\right)<A\left(S_{i}\right)-c_{n} v^{(n-1) / n}+\left|H_{0}\right| v+(C / 2) v^{2}<A\left(S_{i}\right)
$$

for small $v$ and hence for large $i$. Then

$$
F\left(T_{i}\right)<A\left(S_{i}\right)<A\left(S_{0}\right)=F\left(S_{0}\right),
$$

a contradiction of the fact that $S_{0}$ minimizes $F$ in $U$.

REMARK 3 For minimal surfaces, the result also holds without volume constraints. The same proof holds, with simplifications.

REMARK 4 When the ambient manifold $M$ has nontrivial isometries, it suffices to assume that $S_{0}$ has positive second variation orthogonal to the isometries, for fixed volume. Our same proof applies because [W] Thm. 3] immediately generalizes. White observes that a sequence of other minimizers in shrinking physical neighborhoods of $S$ are almost minimizing and hence Hölder differentiable manifolds that converge Hölder differentiably to $S$, contradicting the positive second variation of $S$. In the presence of isometries, one may translate the nearby minimizers to be graphs of functions orthogonal to the isometries to obtain the same contradiction.

As a direct consequence of [CS, Prop. 3.2], Theorem 2, and Remark 4, we have the following:

Corollary 5 In a flat torus $T^{n}$ of dimension $n \leqslant 7$, let $S_{0}$ be a smooth oriented constant-meancurvature hypersurface, possibly with boundary, with positive second variation orthogonal to any isometries of $M$ for fixed volume and boundary. Then for some $\varepsilon_{0}>0$, for $0<\varepsilon<\varepsilon_{0}$, there is a family $u_{\varepsilon}$ of $L^{1}$-local minimizers of the Cahn-Hilliard energy $E_{\varepsilon}$ converging in $L^{1}$ to $S_{0}$.

In particular, as Ross [Ro] proved that the $P, D$, and $G$ minimal surfaces in $T^{3}$ have positive second variation orthogonal to the isometries of $T^{3}$, it follows that there are $L^{1}$-nearby diffuseinterface local minimizers of the Cahn-Hilliard energy. 


\section{Acknowledgements}

We are grateful to Rustum Choksi and Peter Sternberg for calling our attention to this problem and to Robert Kohn for helpful conversations.

The first author is partially supported by a National Science Foundation grant and the Spanish Ministerio de Ciencia y Tecnología FEDER grant MTM2007-61919. The second author is partially supported by MCyT-FEDER MTM2007-61775 and J. Andalucía P06-FQM-01642 grants

\section{REFERENCES}

[A] Allard, W. K. On the first variation of a varifold. Ann. of Math. (2) 95 (1962), 417-491. Zbl 0252.49028 MR 0307015

[B] BRaides, A. $\Gamma$-convergence for Beginners. Oxford Univ. Press, New York (2002). Zbl pre01865939 MR 1968440

[C] Cheung, L.-F. A non-existence theorem for stable constant mean curvature hypersurfaces. Manuscripta Math. 70 (1991), 219-226. Zbl 0734.53011 MR 1085634

[CS] Choksi, R., \& Sternberg, P. Periodic phase separation: the periodic isoperimetric and CahnHilliard problems. Interfaces Free Bound. 8 (2006), 371-392. Zbl 1109.35092 2273234

[dS] DA SilveIRA, A. Stability of complete noncompact surfaces with constant mean curvature. Math. Ann. 277 (1987), 629-638. Zbl 0627.53045 MR 0901709

[F] Fleming, W. H. On the oriented Plateau problem. Rend. Circ. Mat. Palermo 11 (1962), 69-90. Zbl 0107.31304 MR 0157263

[G] Giusti, E. Minimal Surfaces and Functions of Bounded Variation. Birkhäuser, Boston (1984) Zbl 0545.49018 MR 0775682

[Gr] Grosse-Brauckmann, K. Stable constant mean curvature surfaces minimize area. Pacific J. Math. 175 (1996), 527-534. Zbl 0878.49026 MR 1432843

[KS] Kohn, R. V., \& Sternberg, P. Local minimizers and singular perturbations. Proc. Roy. Soc. Edinburgh Sect. A 111 (1989), 69-94. Zbl 0676.49011 MR 0985990

[LR] López, F. J., \& Ros, A. Complete minimal surfaces with index one and stable constant mean curvature surfaces. Comment. Math. Helv. 64 (1989), 34-43. Zbl 0679.53047 MR 0982560

[M1] Morgan, F. Geometric Measure Theory: a Beginner's Guide. 4th ed., Academic Press (2009). Zbl 1179.49050 MR 2455580

[M2] Morgan, F. Regularity of isoperimetric hypersurfaces in Riemannian manifolds. Trans. Amer. Math. Soc. 355 (2003), 5041-5052. Zbl 1063.49031 MR 1997594

[PR] PACARD, F., \& RitorÉ, M. From constant mean curvature hypersurfaces to the gradient mean curvature theory of phase transitions. J. Differential Geom. 64 (2003), 359-423. Zbl 1070.58014 MR 2032110

[R1] Ros, A. The isoperimetric problem. In: Global Theory of Minimal Surfaces, Clay Math. Proc. 2, Amer. Math. Soc., Providence, RI (2005), 175-209. Zbl 1125.49034 MR 2167260

[R2] Ros, A. Stable periodic constant mean curvature surfaces and mesoscopic phase separation. Interfaces Free Bound. 9 (2007), 355-365. Zbl 1142.53013 MR 2341847

[Ro] Ross, M. Schwarz $P$ and $D$ surfaces are stable. Differential Geom. Appl. 2 (1992), 179-195. Zbl 0747.53010 MR 1245555

[W] White, B. A strong minimax property of nondegenerate minimal submanifolds. J. Reine Angew. Math. 457 (1994), 203-218. Zbl 0808.49037 MR 1305283 